\title{
Un nuovo ed efficace attrattivo per la cattura di Drosophila Suzukii basato su ceppi di oenococcus oeni
}

\author{
Raffaele Guzzon, Gianfranco Anfora, Alberto Grassi, e Claudio Ioriatti \\ Fondazione Edmund Mach, via E. Mach 1, 38121 San Michele all’Adige (TN), Italy
}

\begin{abstract}
Sintesi. Drosophila suzukii (SWD) è un insetto parassita di grande rilevanza per la vite. Tra le strategie di contrasto le esche attrattive sono uno strumento fondamentale per un controllo efficiente e sostenibile di questo parassita. Recentemente gli autori hanno sviluppato un'esca alimentare, chiamata Droskidrink, in grado di garantire buone catture di SWD. Tuttavia nuovi e più efficienti strumenti sono necessari per il controllo di questo parassita. Questo lavoro prende avvio dallo studio delle caratteristiche chimiche e microbiologiche del Droskidrink, in relazione ai meccanismi di attrazione verso SWD. È stato così dimostrato che metaboliti caratteristici di fermentazioni batteriche rivestono un fondamentale ruolo nell'attrazione verso SWD. E' stata quindi stata sperimentata l'aggiunta di ceppi batterici all'esca per incrementarne l'attrattività. Trappole innescate con Droskidrink e inoculate con batteri lattici sono state testate nel 2014 in provincia di Trento. Le esche fermentate sono state caratterizzate mediante GC-MS per identificare il profilo dei composti volatili emessi dai batteri. Gli estratti ottenuti dallo spazio di testa di ogni esca sono stati utilizzati per un'analisi elettroantennografica, registrando la risposta delle antenne di SWD. Tutti i risultati concordano nell'individuare in 3 ceppi di Oеnосисcus oeni i batteri più attrattivi verso SWD. Questo lavoro fornisce nuove conoscenze nella biologia di SWD e informazioni utili per la produzione efficaci esche per la cattura di questo insetto.
\end{abstract}

\section{Introduzione}

Drosophila suzukii (SWD) è un insetto nativo del Sud-Est asiatico, dove è stato identificato nel 1931. Importato in Occidente, SWD provoca oggi gravi perdite in importanti produzioni frutticole tra le quali la vite. Aggressività e adattabilità a molti contesti rendono SWD uno dei principali parassiti in agricoltura, in grado di causare perdite di produzione superiori al 90\%. Ad oggi il contrasto a SWD è condotto attraverso l'uso di insetticidi, la cattura massale o pratiche agronomiche specifiche ma nessuno di questi metodi ha portato ad una soluzione del problema.

Precedenti lavori hanno indagato il comportamento di SWD, dimostrando il ruolo che i composti volatili percepiti dai sistemi olfattivi dell'insetto giocano nel determinarne il comportamento [1-3]. Studi condotti sul genere Drosophila hanno chiarito il contributo dei metaboliti rilasciati dal microbiota che colonizza i substrati frequentati dagli insetti. Sono invece scarse le informazioni sull'ecologia di SWD. Quest'insetto è attratto, per la nutrizione, da composti fermentati ma depone le uova solo su frutti sani, utilizzando il suo ovopositore seghettato [4]. Pertanto, le conoscenze acquisite sul insetto modello D. melanogaster, non sono in grado di fornire informazioni esaustive per comprendere il comportamento di SWD.

Metodi di controllo basati su trappole attrattive sono tra le strategie più promettenti per contrastare SWD. Test effettuati confrontando l'attrattività di miscele di etanolo e acido acetico con quella di esche a base di vino e aceto suggeriscono che composti secondari di origine fermentativa possano svolgere un ruolo chiave nella attrazione di SWD [5]. Queste osservazioni confermano l'importanza dell' attività microbica per la definizione della traccia olfattiva utilizzata da SWD per il riconoscimento dei siti di alimentazione e ovideposizione.

Il presente lavoro parte dalla scoperta della sensibilità SWD per alcune molecole prodotte dai batteri lattici come metaboliti secondari della degradazione del glucosio o dell'acido malico, tra cui l'acetoino e il diacetile. Queste molecole non sono comunemente presenti negli attrattivi commerciali per la cattura di SWD. In questo lavoro è quindi descritta un nuovo trappola per catturare SWD caratterizzata dalla presenza di una specifica miscela attraente e dall'aggiunta di colture vitali di batteri lattici.

\section{Materiali e metodi}

\subsection{Microrganismi e analisi microbiologiche}

Diversi ceppi di batteri lattici, appartenenti ai generi Oenococcus, Pediococcus, e Lactobacillus sono stati considerati. I batteri sono stati coltivati su MRS Broth (Oxoid, UK) e mantenuti a $-80^{\circ} \mathrm{C}$ prima dell'uso. La quantificazione della concentrazione batterica è stata svolta mediante conta su piastra, utilizzando MRS Agar (Oxoid) modificato aggiungendo il $15 \% \mathrm{v} / \mathrm{v}$ di succo di mela, secondo i metodi OIV [6].

This is an Open Access article distributed under the terms of the Creative Commons Attribution License 4.0, which permits unrestricted use, distribution, and reproduction in any medium, provided the original work is properly cited. 


\subsection{Composizione della miscela attrattiva}

La miscela, denominata Droskidrink [5], è composta da $75 \%$ di aceto di mele (Prantil, I), 25\% di vino rosso (Caviro, I) e $5 \mathrm{~g} / \mathrm{L}$ di zucchero di canna. Due esche commerciali, Pherocon SWD (Trece, USA) e Droskidrink Prantil (I) sono stati utilizzati come riferimenti nelle prove in campo.

\subsection{Allevamento degli insetti}

Esemplari femmina di SWD, accoppiati, sono stati utilizzati per gli esperimenti. Gli insetti sono stati prodotti nell'allevamento della Fondazione Mach. Le condizioni di allevamento prevedono un intervallo di temperatura tra i $26^{\circ} \mathrm{C}$ (diurna) e i $20^{\circ} \mathrm{C}$ (notturna), umidità del $65 \pm$ $5 \%, 16 \mathrm{~h}$ di luce e $8 \mathrm{~h}$ di buio. Gli insetti sono stati alimentati con una dieta composta da: zucchero ( $15 \mathrm{~g})$, soia $(10 \mathrm{~g})$, lievito $(17 \mathrm{~g})$, farina di mais $(71 \mathrm{~g})$, agar $(5,6 \mathrm{~g})$, multivitaminico $(2,5 \mathrm{~g})$, proprionil $(4,7 \mathrm{ml})$.

\subsection{Analisi di composti volatili del Droskidrink mediante GC-MS e analisi elettroantennografica (EAG)}

La caratterizzazione dei composti volatili è stata eseguita mediante analisi GC-MS dello spazio di in campioni di Droskidrink inoculati con differenti ceppi batterici. $5 \mathrm{ml}$ di campione sono stati posti in fiale ermetiche in vetro $(20 \mathrm{ml})$ e campionati automaticamente (Multipurpose Sampler MPS, Gerstel, D). Ogni fiala è stata incubata a $38^{\circ} \mathrm{C}$ per $20 \mathrm{~min}$ prima del prelievo $(1 \mathrm{ml})$ svolto con siringa riscaldata a $80^{\circ} \mathrm{C}$. L'analisi GC-MS è stata eseguita con un gascromatografo 7890A (Agilent Technologies, USA), equipaggiato con colonna HP-5ms (Agilent Technologies) e accoppiato con un detector di massa XL5975 (Agilent Technologies). Come gas di trasporto è stato utilizzato elio (portata di 1,2 $\mathrm{ml} / \mathrm{min}$ ); il ciclo termico ha preveisto: $5 \mathrm{~min}$ a $30^{\circ} \mathrm{C}$, una rampa di temperatura di $3,5^{\circ} \mathrm{C} / \mathrm{min}$ fino al $240^{\circ} \mathrm{C}$ e $2 \mathrm{~min}$ a $240^{\circ} \mathrm{C}$. L'acquisizione e l'analisi dei dati è stata fatta con il software ChemStation (Agilent Technologies). Le miscele fermentate sono state anche utilizzate per l'analisi EAG su femmine accoppiate di SWD $(\mathrm{n}=5)$ [7].

\subsection{Test in campo}

Per le prove in campo sono state utilizzate trappole in plastica rossa (Droso-TRAP, Biobest, B), riempite con $200 \mathrm{ml}$ di Droskidrink contenente batteri. La concentrazione batterica nel Droskidrink all'inizio dei test in campo è stata regolata a $10^{6} \mathrm{ufc} / \mathrm{ml}$. Le trappole sono state collocate in modo casuale lungo i filari dei vigneti (ogni $2 \mathrm{mt}$, altezza dal suolo 1,5 mt) e la posizione delle trappole è stata modificata in modo casuale ogni settimana. Dopo 1 settimana di esposizione dell'esca il liquido è stato filtrato e inviato alle analisi. Gli insetti catturati sono stati identificati con osservazione diretta utilizzando uno stereoscopio (Optika, I).

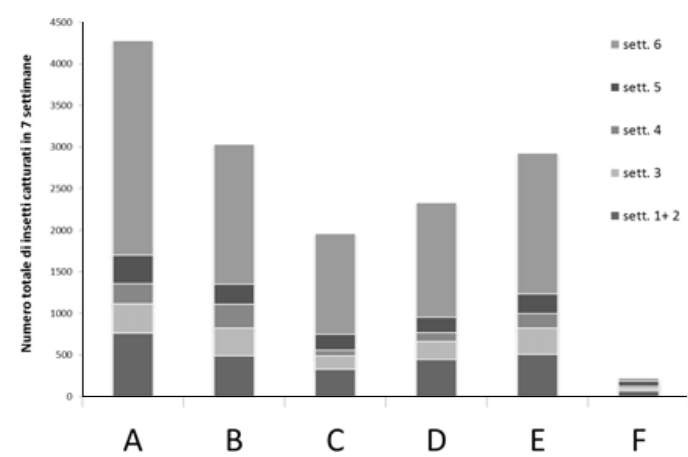

Figura 1. Somma delle catture di SWD svolte in 6 settimane da trappole con diversi ceppi batterici. A $O$. oeni, B Pediococcus pentosaceous, C, D, E differenti ceppi di Lactobacillus, F testimone senza batteri.

\section{Risultati e discussione}

\subsection{Test di attrattività verso SWD di diverse specie batteriche}

Le prime prove in campo sono state svolte per testare l'effetto dell'aggiunta di batteri lattici all'esca attrattiva. In Figura 1 sono riassunti i risultati di una campagna di catture di SWD di 6 settimane. I diversi batteri testati sono A) O. oeni, B) P. pentosaceus. C, D, E) diversi ceppi di Lactobacillus, F) un'esca commerciale non inoculata con batteri. I risultati dei test di cattura dimostrano l'elevata attività di $O$. oeni sia rispetto ad altri generi batterici che in confronto con l'attrattivo commerciale. Per quanto riguarda i generi batterici testati, è noto che Oenococcus e Pediocccus abbiano una notevole attività etero fermentante $[8,9]$ con produzione di diacetile e acetoino, pertanto la loro elevata efficacia nella cattura di SWD non è inaspettata. Lo scarso rendimento della tesi F conferma il ruolo fondamentale svolto dai microrganismi nella attrazione di SWD. Queste prime evidenze inducono a considerare $O$. oeni la specie più promettente tra $\mathrm{i}$ batteri lattici testati, ulteriori test sono però necessari per chiarire il comportamento di questi batteri all'interno della trappola.

\subsection{Caratterizzazione GC-MS del profilo dei volatili prodotti da diversi ceppi di O. oeni dopo la fermentazione del Droskidrink}

L'analisi GC-MS ha determinato il profilo dei composti volatili rilasciati dal Droskidrink inculato con diversi ceppi di batteri appartenenti alla specie $O$. oeni. Sono stati presi in considerazione 3 ceppi con particolari caratteristiche di resistenza alla composizione chimica del Droskidrink (dati non riportati), confrontati con un ceppo di riferimento della stessa specie. Nella Tabella 1 è riportata la produzione relativa di composti volatili, rispetto a quanto presente in un Droskidrink sterile, per ciascuno dei ceppi di $O$. oeni. Sono stati rilevati 13 composti (Tabella 1). Il ceppo G1 è caratterizzato da una elevata produzione di acido acetico ed etanolo. I ceppi G2 e G3 mostrato una notevole riduzione del contenuto di acido acetico, mentre l'etanolo aumenta del $30 \%$. Il contenuto di 3-metil-1-butanolo, riconosciuto come attrattivo "nontarget" per una vasta gamma di insetti [10], è superiore alla 
Tabella 1. Composizione relativa in termini di molecole volatili dei Droskidrink dopo inoculo batterico. Dati espressi in contenuto relativo (\%) rispetto al Droskidrink sterile.

\begin{tabular}{lcccc}
\hline \multirow{2}{*}{ Composto volatile } & \multicolumn{4}{c}{ Ceppi di O. oeni } \\
\cline { 2 - 5 } Ac. acetico & Type & G1 & G2 & G3 \\
Etanolo & -52.2 & +560.0 & -44.1 & -44.3 \\
3-metil-1-butanolo & +26.1 & +54.7 & +38.5 & +29.3 \\
\hline Alcool Isoamilico & +23.7 & +0.9 & +70.0 & +21.1 \\
Acetoino & +49.4 & -55.4 & +88.1 & +36.7 \\
\hline Acetidno & -95.0 & -96.1 & -93.0 & +69.2 \\
2-butil acetato & +14.6 & -31.3 & +12.5 & +5.5 \\
\hline Acetato di isobutile & -29.3 & -56.2 & +6.8 & -30.8 \\
Butirrato di etile & -35.1 & -48.3 & -9.2 & -37.8 \\
\hline Acetato di isoamile & -59.8 & -70.5 & -57.8 & -62.8 \\
Caproato di etile & -4.2 & -49.4 & +47.4 & -7.53 \\
Octanoato di etile & +43.4 & -27.4 & +141.4 & +35.6 \\
2-butanone & +39.4 & -15.0 & +64.7 & +49.3 \\
\hline
\end{tabular}

norma nei campioni G2 e G3. L'acetoino, molecola chiave per l'attrazione di SWD, mostra una riduzione del $55 \%$ nel ceppo G1, mentre la sua concentrazione è elevata nel caso dei ceppi G2 e G3. Il 2-butanone ha un andamento simile. Nel caso degli esteri l'attività batterica è più uniforme con una riduzione generalizzata del contenuto iniziale e due sole eccezioni nel campione G3 che ha registrato un aumento del 2-butil acetato e dell'ottanoato di etile. I risultati dell'analisi GC-MS ci restituiscono quindi un quadro compositivo complesso, dove la fermentazione muta il profilo dei composti volatili delle esche. È necessario tener conto del fatto che una concentrazione eccessiva di alcuni composti volatili può indurre una risposta repulsiva nel sistema olfattivo dell'insetto [11]. Pertanto tutte le variazioni quantitative indotte dai batteri devono essere considerate con attenzione, in relazione all'ambiente (tempo, temperatura) nel quale la trappola è esposta. In ogni caso, è stata osservata una differenza significativa tra i diversi ceppi di $O$. oeni, differenza che richiede nuove prove in campo per individuare la migliore miscela attrattiva.

\subsection{Analisi EAG dei Droskidrink fermentati}

I risultati dell'analisi EAG sono riportati in Fig. 2. I composti di controllo, 1-esanolo e 2-esanale sono fortemente percepiti dagli insetti, come previsto. Non sono state rilevate differenze statistiche tra i fermentati dei ceppi TYPE, G1 e G3 mentre il campione G2 ha mostrato una risposta più bassa, inferiore a quella del bianco, ovvero il Droskidrink non inculato. L'analisi conferma quindi l'azione dei ceppi batterici nel modulare la risposta di SWD, probabilmente un'analisi EAG accoppiata all'identificazione gascromatografica dei composti in grado di eccitare le antenne di SWD potrebbe meglio chiarire quali molecole volati siano particolarmente significative per questo insetto.

\subsection{Test di cattura in campo con trappole innescate con diversi ceppi di 0 . oeni}

Due prove in campo sono state eseguite per valutare l'effetto dell'aggiunta di batteri alle trappole, in termini

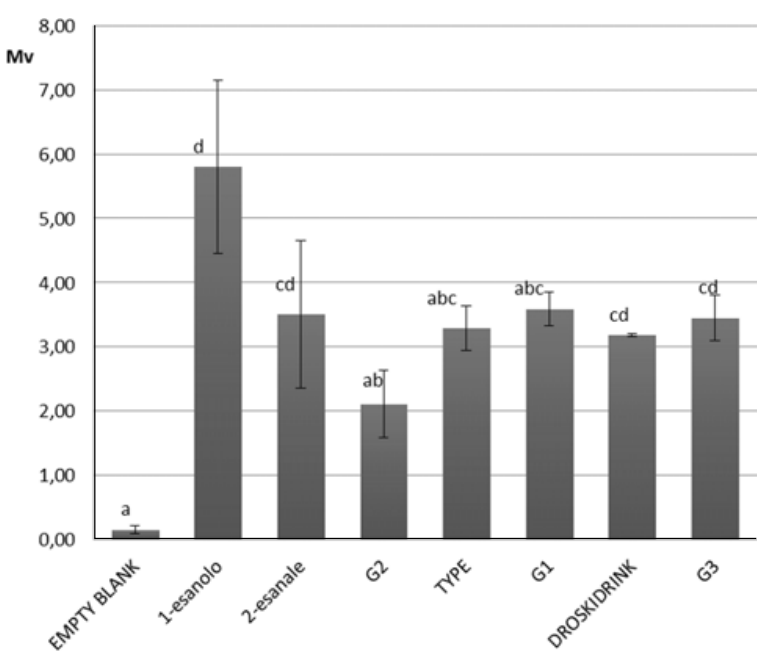

Figura 2. Risposte all'analisi EAG su esemplari di SWD dei diversi Droskidrink fermentati da batteri lattici appartenenti alla specie $O$. oeni.

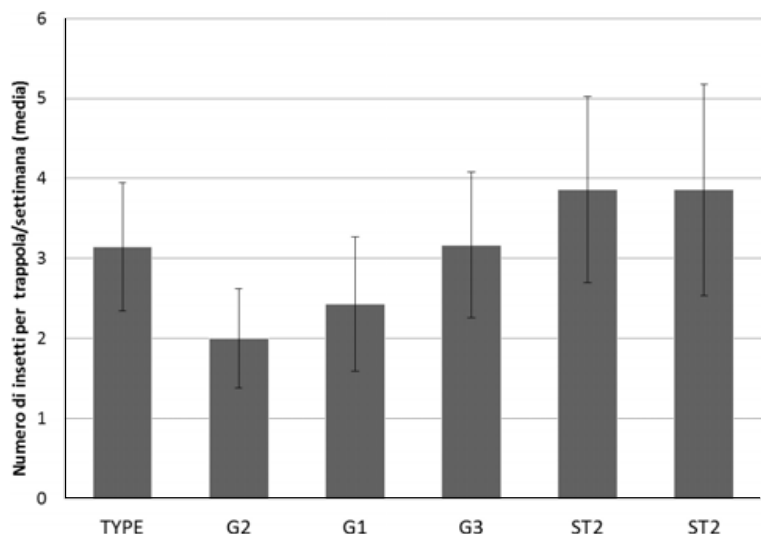

Figura 3. Catture di esemplari di SWD in prove in campo svolte ad inizio primavera 2014 (5 settimane), utilizzando esche innescate con diversi ceppi di $O$. oeni. G1, G2, G3, Type: ceppi di O. oeni. ST1 e ST2, attrattivi commerciali.

di catture di SWD. Il primo test è condotto all'inizio della primavera 2014, il secondo test durante l'estate dello stesso anno. In Figs. 3 e 4 sono riportati i risultati delle catture per ogni tipo di esca. Il primo esperimento è stato caratterizzato da catture molto basse e nessuna differenza statisticamente significativa tra le diverse tesi sperimentali (Fig. 3). La scarsa attività delle esche può essere dovuta alle basse temperature ambientali che hanno ridotto l'attività batterica [12] e l'evaporazione delle sostanze volatili attrattive. Tuttavia, dissezioni delle femmine adulte catturate durante questo primo esperimento hanno permesso di osservare che circa 1' $80 \%$ degli insetti conteneva completamente uova sviluppato, supportando l'ipotesi che le femmine di SWD svernino dopo l'accoppiamento. Una cattura massale precoce può quindi essere una efficace strategia per ridurre la popolazione e prevenire massicci attacchi alla ripresa vegetativa.

Il secondo esperimento campo è stato eseguito in estate, per 7 settimane consecutive (Fig. 4). In questo caso le temperature erano adeguate ad una rapida crescita 


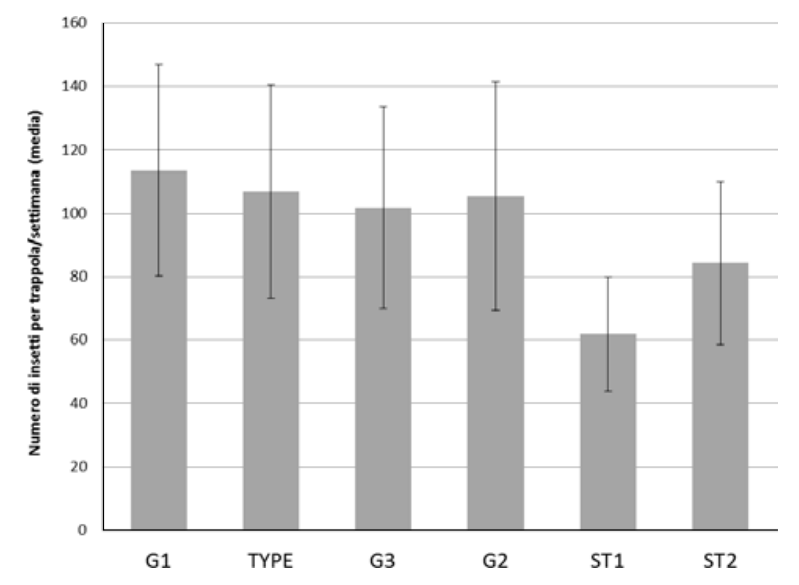

Figura 4. Catture di esemplari di SWD in prove in campo svolte nell'estate 2014 (7 settimane), utilizzando esche innescate con diversi ceppi di O. oeni. G1, G2, G3, Type: ceppi di O. oeni. ST1 e ST2, attrattivi commerciali.

batterica ed infatti è stato possibile osservare catture massicce con punte di 3000 individui a settimana. Sono anche evidenti differenze nell'attrattività delle differenti esche. In particolare l'esca inoculato con il ceppo G3 ha mostrato le migliori prestazioni.

\section{Conclusioni}

La cattura massale si è rivelata a una efficace strategia per il contenimento di SWD. Tuttavia le trappole attualmente in commercio non sembrano garantire una efficacia tale da rappresentare una effettiva soluzione al problema. Trappole attivate con specifici ceppi di batteri, in grado di ottimizzare la produzione di volatili particolarmente attrattivi per SWD possono rappresentare una interessante alternativa in grado di ottimizzare le catture massali di questo pericoloso parassita.

\section{Bibliografia}

[1] T.J.A. Bruce, J.A. Pickett. Phytochem. 72, 1605-1611 (2011)

[2] M. Hilker, J. McNeil. Chapter 5 in E. Wajnberg, C. Bernstein, J. van Alphen eds. Behavioral Ecology of Insect Parasitoids: From Theoretical Approaches to Field Applications. Blackwell

[3] T.J.A. Bruc, L.J. Wadhams, C.M. Woodcock. Trends Plant Sci. 10, 269-74 (2005)

[4] A. Cini, C. Ioriatti, G. Anfora. Bull. Insectol. 65, 149-160 (2012)

[5] D.H. Cha, S.P. Hesler, R.S. Cowles, H. Vogt, G.M. Loeb, P.J. Landolt. Environ. Entomol. 42, 1052-1060 (2013)

[6] OIV. Recueil international des méthodes d'analyses. Analyse microbiologique des vins et des moûts MA-AS4-01 (2015)

[7] M. Tasin, G. Anfora, C. Ioriatti, A. Cristofaro, S. Carlin, A. De Cristofaro, S. Schmidt, M. Bengtsson, G. Versini, Witzgall P. J. Chem. Ecol. 31, 77-87 (2005)

[8] S.Q. Liu, SQ. J. App. Microbiol. 92, 589-601 (2002)

[9] A. Lonvaud-Funel. in A.G. Reynolds. Managing wine quality, Vol 2. Woodhead Publishing Series in Food Sci. Technol. Nut. 192, 60-92 (2010)

[10] P.J. Landolt, T. Adams, H. Rogg. J. App. Entomol. 136,148-154 (2012)

[11] D.H. Cha, T. Adams, H. Rogg, P.J. Landolt. J. Chem. Ecol. 38, 1419-1431 (2012)

[12] R. Guzzon, E. Poznanski, L. Conterno, P. Vagnoli, S. Krieger-Weber, A. Cavazza. S. Af. J. Enol. Vitic. 30, 41-49 (2009) 